Article

\title{
Identification and Characterization of Quorum-Quenching Activity of $N$-Acylhomoserine Lactonase from Coagulase-Negative Staphylococci
}

\author{
Tomohiro Morohoshi ${ }^{1, *(1)}$, Yaoki Kamimura ${ }^{1}$ and Nobutaka Someya ${ }^{2}$ \\ 1 Department of Material and Environmental Chemistry, Graduate School of Engineering, Utsunomiya \\ University, Utsunomiya 321-8585, Japan; yaokami@gmail.com \\ 2 Institute of Vegetable and Floriculture Science, National Agriculture and Food Research Organization, \\ 3-1-1 Kannondai, Ibaraki 305-8519, Japan; someyan@affrc.go.jp \\ * Correspondence: morohosi@cc.utsunomiya-u.ac.jp
}

Received: 15 July 2020; Accepted: 4 August 2020; Published: 5 August 2020

\begin{abstract}
N-Acylhomoserine lactones (AHLs) are used as quorum-sensing signals in Gram-negative bacteria. Many genes encoding AHL-degrading enzymes have been cloned and characterized in various microorganisms. Coagulase-negative staphylococci (CNS) are present on the skin of animals and are considered low-virulent species. The AHL-lactonase gene homologue, ahlS, was present in the genomes of the CNS strains Staphylococcus carnosus, Staphylococcus haemolyticus, Staphylococcus saprophyticus, and Staphylococcus sciuri. We cloned the candidate ahlS homologue from six CNS strains into the pBBR1MCS5 vector. AhlS from the CNS strains showed a higher degrading activity against AHLs with short acyl chains compared to those with long acyl chains. AhlS from S. sciuri was expressed and purified as a maltose-binding protein (MBP) fusion. Pseudomonas aeruginosa is an opportunistic pathogen that regulates several virulence factors such as elastase and pyocyanin by quorum-sensing systems. When MBP-AhlS was added to the culture of P. aeruginosa PAO1, pyocyanin production and elastase activity were substantially reduced compared to those in untreated PAO1. These results demonstrate that the AHL-degrading activity of AhlS from the CNS strains can inhibit quorum sensing in P. aeruginosa PAO1.
\end{abstract}

Keywords: quorum sensing; $N$-acylhomoserine lactone; coagulase-negative staphylococci; AHL lactonase; Pseudomonas aeruginosa

\section{Introduction}

Quorum sensing is a cell-to-cell communication system used by many bacterial species depending on their population densities [1]. One of the most common quorum-sensing signals, $N$-acyl-L-homoserine lactone (AHL), is used by Gram-negative bacteria [2]. Once the AHL reaches a threshold concentration, the transcription of specific genes is activated, resulting in the expression of phenotypes such as motility, adhesion, biofilm formation, toxicity, and pathogenicity [3]. AHL-negative mutants of many pathogens generally have defected pathogenicity [3,4]. Quorum quenching, which disrupts or manipulates quorum-sensing signals, is one of the most effective techniques to inhibit the expression of virulence and disrupt the infection of host cells. Many AHL-degrading enzymes have been cloned from various microorganisms and studied for their applications in the control of infectious diseases [5]. AHL-degrading enzymes have been divided into two functional groups-AHL lactonase and AHL acylase [5]. AHL lactonase catalyzes AHL ring-opening by hydrolyzing lactones, whereas AHL-acylase hydrolyzes the amide bond of AHL [5]. AiiA-type AHL lactonase, first identified from Bacillus sp. 240B1, is the most-studied AHL-degrading enzyme [6]. It belongs to the metallo- $\beta$-lactamase superfamily and 
has been identified and characterized in various bacteria such as AhlS from Solibacillus silvestris [7], AttM from Agrobacterium tumefaciens [8], AhlD from Arthrobacter sp. [9], and AidC from Chryseobacterium sp. [10]. In addition, we have previously reported a highly-thermostable AiiA-type AHL lactonase, AiiT, which was isolated from the thermophilic bacterium Thermaerobacter marianensis JCM 10246 [11].

Coagulase-negative staphylococci (CNS) are known to comprise over 30 species, which are part of the normal flora on the skin of animals [12]. Coagulase, which converts fibrinogen into fibrin, is a major virulence factor produced by coagulase-positive staphylococci (CPS), e.g., Staphylococcus aureus and Staphylococcus pseudintermedius [12]. Therefore, CNS strains are considered less virulent than CPS strains. Gram-positive bacteria such as CNS strains do not produce any AHL molecules but use oligopeptide as their quorum-sensing signals [1,3]. Meanwhile, some Gram-positive bacteria produce AHL-degradative enzyme and inhibit quorum sensing in other Gram-negative bacteria [5]. So far, AHL-degradative enzymes isolated from CNS strains have not been reported. In this study, we identified and characterized novel AHL-degradative genes from several CNS strains.

\section{Results and Discussion}

\subsection{AHL-Degrading Activities in CNS Strains}

Six strains from four CNS species, Staphylococcus carnosus, Staphylococcus haemolyticus, Staphylococcus saprophyticus, and Staphylococcus sciuri were selected to evaluate their AHL-degrading activities. CNS strains were cultivated in Luria-Bertani (LB) liquid medium containing $20 \mu \mathrm{M}$ $N$-hexanoyl-L-homoserine lactone (C6-HSL) or N-decanoyl-L-homoserine lactone (C10-HSL). After 4.5 and $9 \mathrm{~h}$ incubation, the remaining AHLs in the culture supernatant were visualized by AHL biosensors, Chromobacterium violaceum CV026 (for the short chain C6-HSL) and VIR07 (for the long chain C10-HSL), which produced the purple pigment violacein $[13,14]$. The results of AHL degradation are shown in Figure 1. All CNS strains used in this study completely degraded $20 \mu \mathrm{M}$ C6-HSL by $9 \mathrm{~h}$ incubation, confirming their C6-HSL-degrading activities. Specifically, S. sciuri ATCC 29060 and 29061 completely degraded $20 \mu \mathrm{M}$ C6-HSL by $4.5 \mathrm{~h}$ incubation, suggesting higher AHL-degrading activities than those of other strains. In contrast, most C10-HSL remained by $4.5 \mathrm{~h}$ incubation in the supernatants of S. carnosus, S. haemolyticus, and S. saprophyticus. Lower levels of C10-HSL were detected in the culture supernatants of S. sciuri ATCC 29060 and 29061, which also had higher C6-HSL-degrading activities. After $9 \mathrm{~h}$ incubation, all CNS strains completely degraded $20 \mu \mathrm{M}$ C10-HSL except for S. haemolyticus NBRC 109768. These results suggested that the CNS strains tested in this study had higher degrading activities toward C6-HSL than C10-HSL.

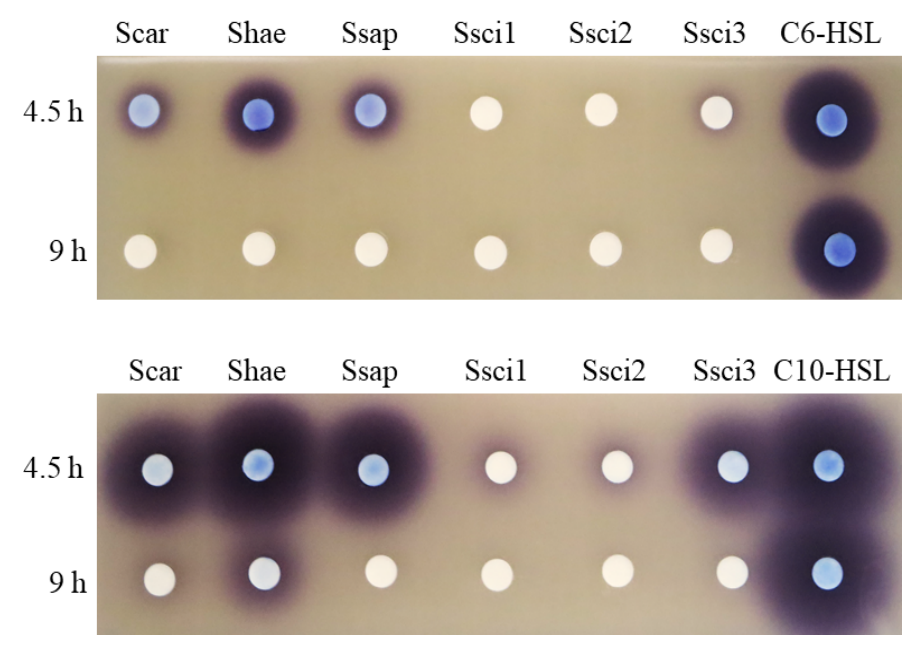

Figure 1. N-Acylhomoserine lactone (AHL)-degrading activities of CNS strains. Full-grown cultures of CNS strains, Staphylococcus carnosus NBRC 109622 (Scar), Staphylococcus haemolyticus NBRC 109768 (Shae), Staphylococcus saprophyticus NBRC 102446 (Ssap), Staphylococcus sciuri American Type Culture Collection (ATCC) 29060 (Ssci1), S. sciuri 29061 (Ssci2), and S. sciuri StLB252 (Ssci3) were mixed with 
$20 \mu \mathrm{M} \mathrm{N}$-hexanoyl-L-homoserine lactone (C6-HSL) or N-decanoyl-L-homoserine lactone (C10-HSL) and incubated at $37^{\circ} \mathrm{C}$ for 4.5 and $9 \mathrm{~h}$. The residual AHLs were detected using Chromobacterium violaceum CV026 (for C6-HSL) or VIR07 (for C10-HSL).

\subsection{AiiA-Type AHL Lactonase Gene Is Present in the CNS Strains}

The whole genome sequences of S. carnosus NBRC 109622, S. haemolyticus NBRC 109768, and S. saprophyticus ATCC 15305 (synonym of NBRC 102446) were deposited in DDBJ/ENA/GenBank under the accession numbers BKAO01000000, BKAY01000000, and CP035294, respectively. Gene homologs encoding the AiiA-type AHL-lactonase were searched in these genome sequences. Based on the Basic Local Alignment Search Tool (BLAST) analysis [15], the deduced amino acid sequences of SCA04_19880 from S. carnosus NBRC 109622, SHA04_07910 from S. haemolyticus NBRC 109768, and EQ030_01160 from S. saprophyticus NBRC 102446 showed similarity to the known AiiA-type AHL lactonases. Specifically, the amino acid sequences of these gene homologs showed higher similarity with AhlS from S. silvestris StLB046 [7] and AiiT from T. marianensis JCM 10246 [11]. Although the genome sequences of S. sciuri ATCC 29060 and 29061 have not been released, the ahlS gene homolog (FPV13_12710) was found in the genome of S. sciuri B9-58B (accession No. CP041879) [16]. Therefore, specific polymerase chain reaction (PCR) primers were designed based on the sequence of FPV13_12710 from S. sciuri B9-58B, and the ahlS homolog genes were successfully amplified by PCR from the genomes of ATCC 29060, ATCC 29061, and StLB252. Sequencing of the three ahlS genes from S. sciuri revealed high similarity in their amino acid sequences (over 98\%). The amino acid sequences of AhlS from the CNS strains were also compared to those of the known AiiA-type AHL lactonases (Figure 2), which showed some well-conserved amino acid residues. Specifically, the zinc-binding "HXHXDH" motifs, which were commonly conserved sequences of the known AiiA-type AHL lactonases [6], were also found in the AhlS from the CNS strains (Figure 2). Interestingly, the AhlS from the CNS strains were phylogenetically related to AhlS from S. silvestris and AiiT from T. marianensis but were slightly distant from the other AiiA-type AHL lactonases (Figure S1). Therefore, these proteins might be classified as members of a new subfamily composed of AiiA-type AHL lactonases.

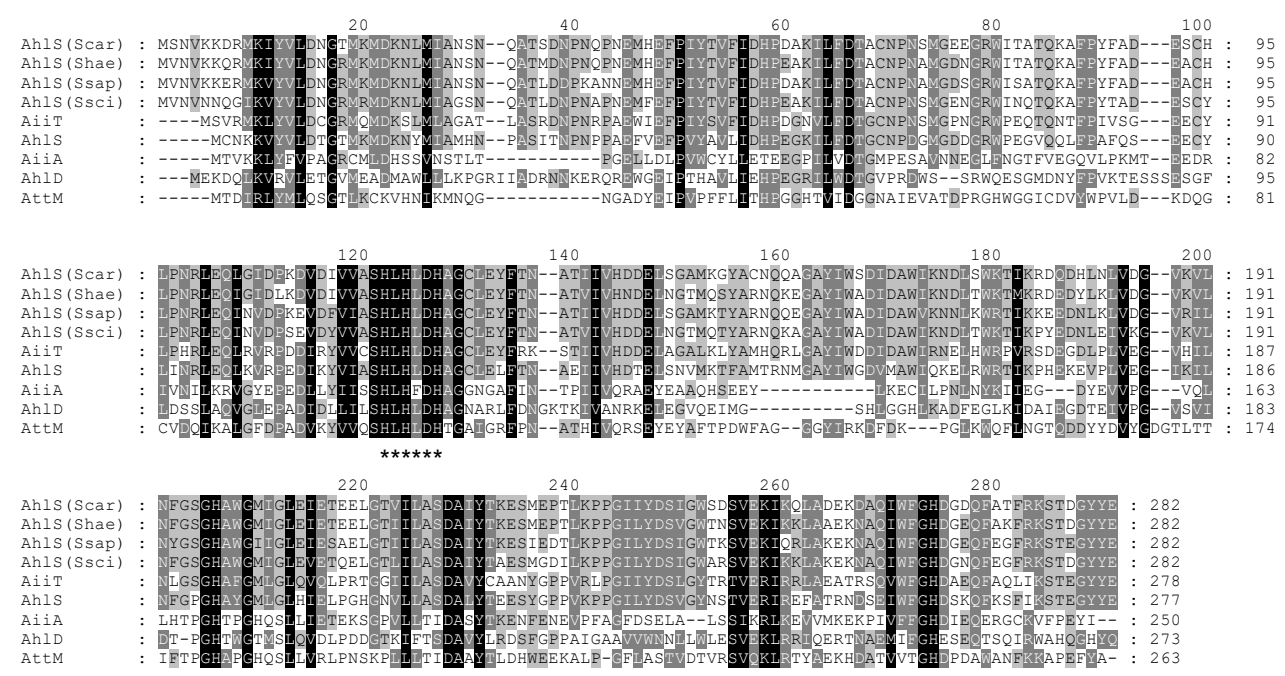

Figure 2. Comparisons of AhlS from the CNS strains with the known AiiA-like AHL lactonases. The amino acid sequences of AhlS from S. carnosus NBRC 109622 (Scar), S. haemolyticus NBRC 109768 (Shae), S. saprophyticus NBRC 102446 (Ssap), and S. sciuri ATCC 29060 (Ssci) were compared to those of the AhlS from Solibacillus silvestris StLB046 (UniProt accession No. F2F233), AiiT from Thermaerobacter marianensis JCM 10246 (E6SI95), AiiA from Bacillus sp. 240B1 (Q9L8R8), AttM from Agrobacterium tumefaciens C58 (Q7D3U0), and Arthrobacter sp. IBN110 (Q7X3T2). Sequences were aligned using ClustalW and shaded using the GeneDoc software. The consensus amino acid sequence (HXHXDH) was marked with asterisks. 


\subsection{Characterization of the AHL-Degrading Activity of AhlS from the CNS Strains}

To test if the AhlS from the CNS strains had AHL-degrading activities, the ahlS genes from the CNS strains were amplified by PCR and cloned into pBBR1MCS5 cloning vectors Escherichia coli $\mathrm{DH} 5 \alpha$, harboring ahlS gene from CNS strains, was grown in the LB medium containing $10 \mu \mathrm{M}$ C6-HSL or C10-HSL. After 3, 6, and $9 \mathrm{~h}$ incubations, the remaining AHLs in the culture supernatants were visualized and quantified by the AHL reporter strains. The results indicated that over $60 \%$ of C6-HSL was degraded by $9 \mathrm{~h}$ incubation by E. coli DH5 $\alpha$ harboring ahlS from the CNS strains (Figure 3A). Specifically, AhlS from S. haemolyticus showed the highest activity by completely degrading C6-HSL after $6 \mathrm{~h}$ incubation. On the other hand, E. coli DH5 $\alpha$ harboring the ahlS from the CNS strains showed slightly lower degrading activity against C10-HSL than C6-HSL (Figure 3B). One of the AHL-degrading enzymes, AHL lactonase, degrades AHLs by hydrolyzing its homoserine lactone ring [5], which can be re-circularized in acidic solutions [17]. Therefore, AHL restoration assay was carried out to test the presence of a putative lactonase activity. The results showed that C10-HSL degraded by E. coli harboring ahlS from the CNS strains was restored by acidification (Figure S2). These results indicated that AhlS from the CNS strains worked as AHL-lactonase, similarly to other AiiA-type AHL lactonases.

A

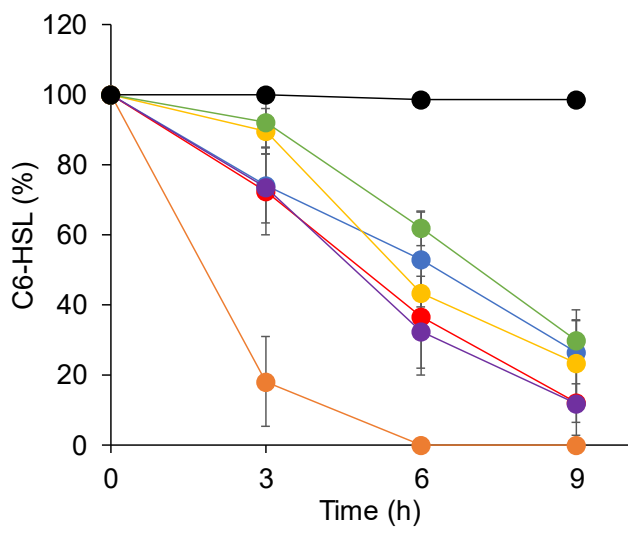

B

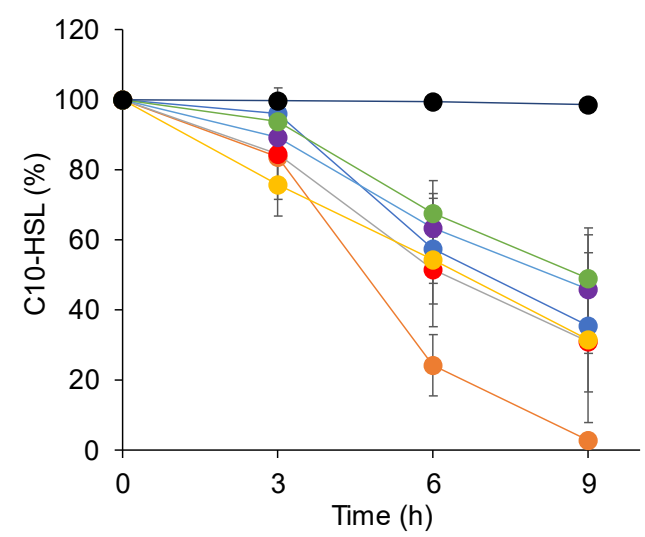

Figure 3. Degradation of C6-HSL (A) and C10-HSL (B) by Escherichia coli DH5 $\alpha$ harboring ahlS genes from the CNS strains. Full-grown cultures of E. coli DH5 $\alpha$ harboring an empty vector pBBR1MCS5 (black) or pBBR1MCS5 containing the ahlS gene from S. carnosus NBRC 109622 (blue), S. haemolyticus NBRC 109768 (orange), S. saprophyticus NBRC 102446 (red), S. sciuri ATCC 29060 (yellow), S. sciuri 29061 (purple), and S. sciuri StLB252 (green) were inoculated into the Luria-Bertani (LB) medium containing $10 \mu \mathrm{M}$ C6-HSL or C10-HSL and incubated at $37^{\circ} \mathrm{C}$ for 3,6 , and $9 \mathrm{~h}$. After incubation, the remaining AHLs in the culture supernatants were visualized by $C$. violaceum and calculated using the relationship equations based on the size of the purple-color zones. The data were reproduced at least three times and the error bars indicate standard deviations.

\subsection{AhlS Inhibits the Quorum-Sensing Phenotypes in Pseudomonas aeruginosa}

To evaluate the potential of AhlS to interfere with quorum sensing in other bacteria, AhlS was expressed and purified as a maltose-binding protein (MBP). First, the ahlS gene from S. sciuri ATCC 29060 was amplified by PCR and cloned into the pMAL-c2x vector. The MBP-AhlS fusion protein was overexpressed in E. coli DH5 $\alpha$ and purified by maltose affinity chromatography. Sodium dodecyl sulfate-polyacrylamide gel electrophoresis (SDS-PAGE) analysis revealed that the overexpressed protein was approximately $75 \mathrm{kDa}$ in size (Figure S3), which was congruent with the predicted molecular weight of MBP-AhlS based on its amino acid sequence. To assess the AHL-degrading activity, MBP-AhlS was mixed with $20 \mu \mathrm{M}$ C6-HSL and C10-HSL and incubated for $30 \mathrm{~min}$ at $37^{\circ} \mathrm{C}$. The results showed that MBP-AhlS completely degraded C6-HSL and C10-HSL within 30 min, whereas MBP-LacZ $\alpha$ as a negative control did not (Figure $4 \mathrm{~A}$ ). 
P. aeruginosa is an opportunistic pathogen that uses the quorum-sensing system to regulate several virulence factors [18]. In P. aeruginosa PAO1, LasI and RhlI are responsible for the synthesis of the las and rhl signals, $\mathrm{N}$-(3-oxododecanoyl)-L-homoserine lactone (3-oxo-C12-HSL) and N-butyryl-L-homoserine lactone (C4-HSL), respectively [18]. Pyocyanin, which is a redox-active phenazine pigment and induces cytotoxicity in human cells [19], is produced by P. aeruginosa in response to AHL-mediated quorum sensing [18]. Elastase, a major virulence factor in P. aeruginosa that degrades tissue matrix proteins in the human host, is also regulated by the quorum-sensing system [18]. Pyocyanin production and elastase activity were drastically reduced in the lasI rhlI double mutant P. aeruginosa PAO-MW1 (Figure 4B,C). Assessment of the effect of MBP-AhlS on quorum sensing in P. aeruginosa showed that the pyocyanin production by P. aeruginosa PAO1 treated with MBP-AhlS was reduced to less than $50 \%$ of that with the control MBP-LacZ $\alpha$ (Figure 4B). The elastase activity in P. aeruginosa PAO1 treated with MBP-AhlS was reduced to $60 \%$ of that with MBP-LacZ $\alpha$ (Figure $4 \mathrm{C}$ ). These results demonstrated that treatment by AhlS contributed to reducing the production of several virulence factors regulated by AHL-mediated quorum sensing in P. aeruginosa.

A

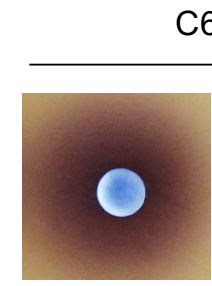

MBP-LacZa
C6-HSL

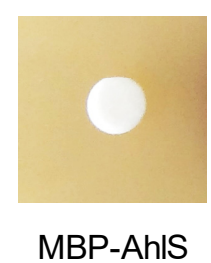

B

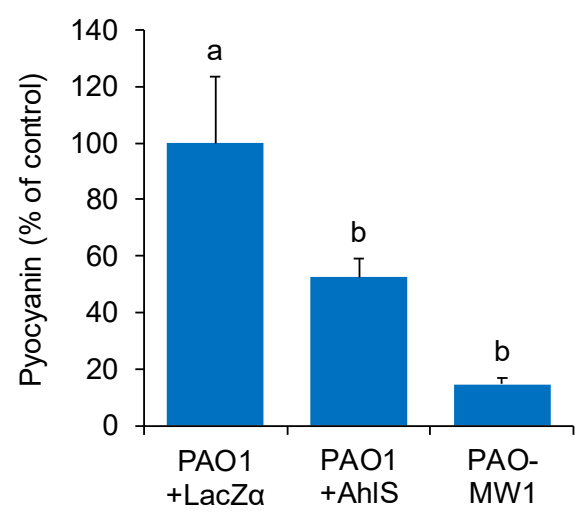

C10-HSL

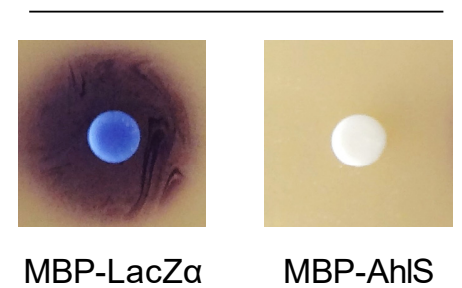

C

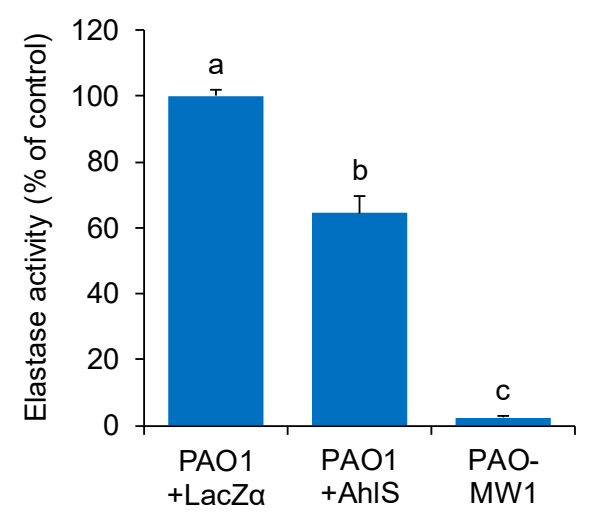

Figure 4. (A) AHL-degrading activities of MBP-LacZ $\alpha$ and MBP-AhlS. Purified proteins were mixed with an equal volume of $20 \mu \mathrm{M}$ C6-HSL or C10-HSL solutions and incubated at $37^{\circ} \mathrm{C}$ for $30 \mathrm{~min}$. The residual AHL was detected using C. violaceum CV026 or VIR07, respectively. Pyocyanin production (B) and elastase activity (C) in culture supernatants of Pseudomonas aeruginosa PAO1 treated with MBP-LacZ $\alpha$ or MBP-AhlS. PAO-MW1 was used as the quorum-sensing-negative control. The average value of PAO1-LacZ $\alpha$ was defined as $100 \%$. The data were reproduced at least three times and the error bars indicate standard deviations. Different lowercase letters indicate significant differences, as determined by the Tukey's HSD test $(p<0.05)$.

\section{Materials and Methods}

\subsection{Bacterial Strains, Compounds, and Incubation Conditions}

S. carnosus NBRC $109622^{\mathrm{T}}$ [20], S. haemolyticus NBRC $109768^{\mathrm{T}}$ [21], and S. saprophyticus NBRC $102446^{\mathrm{T}}$ [21] were obtained from the NITE Biological Resource Center (NBRC), Japan. S. sciuri ATCC 29060 and 29061 [22] were obtained from the American Type Culture Collection (ATCC). S. sciuri 
StLB252 was isolated from the surface of the potato leaf in our unpublished experiment. Bacterial strains were incubated at $37^{\circ} \mathrm{C}$ in the Luria-Bertani (LB) medium [23]. Solid bacterial media were made by adding agar at a final concentration of $1.5 \%$. Ampicillin and gentamycin were added at final concentrations of $100 \mu \mathrm{g} / \mathrm{mL}$ and $10 \mu \mathrm{g} / \mathrm{mL}$, respectively. The C6-HSL and C10-HSL used in this study were synthesized by previously-described methods [24].

\subsection{Amplification and Sequencing of the ahlS Gene from S. sciuri}

To amplify the ahlS gene from the genome of $S$. sciuri, specific PCR primers sciseq-F ( $5^{\prime}$-TTT TAT CGG CCT GGT AAT CCC GTT TTC GGG TCG C-3') and sciseq-R (5'-TTC ATG CCC AAG TGC CAT AGG AGT AGG GC-3') were designed based on the sequence of FPV13_12710 from S. sciuri B9-58B. PCR was performed with the KOD FX Neo system (Toyobo, Osaka, Japan) using the following cycling parameters: $98^{\circ} \mathrm{C}$ for $10 \mathrm{~s}, 60^{\circ} \mathrm{C}$ for $30 \mathrm{~s}$, and $68^{\circ} \mathrm{C}$ for $1.5 \mathrm{~min}$ for 30 cycles. PCR fragments were sequenced using a BigDye Terminator ver. 3.1 sequencing kit and an ABI 3500 Genetic Analyzer (Applied Biosystems, Tokyo, Japan).

\subsection{Cloning of the ahlS Gene from the CNS Strains}

The ahlS coding regions in the genomes of the CNS strains were amplified with the KOD FX Neo system using the following primer sets: car-F (5'-TCT GTC GAC ACC CTC AGT TGT AAT AAG CCA ATA CC-3') and car-R (5'-TCT CTG CAG AAG TCA GAA TGA CAT AAT CCT GCT GC-3') for ahls from $S$. carnosus, hae-F (5'-TCT GTC GAC AAA GCA CAT AAA TAG AGC CTT CC) and hae-R (5'-TCT CTG CAG ACT GAT AAG TCT GAA TGG CAT ATC CC-3') for ahls from S. haemolyticus, sap-F (5'-TCT GTC GAC CTA AAA ATG ACA ACT TTG GAG GGC GC-3') and sap-R (5'-TCT CTG CAG TTT ACC CAT TTC ACG CAA TAC TGC CG-3') for ahls from $S$. saprophyticus, and sci-F (5'-TCT GTC GAC TCG GTC CAT ATT CCA ACC TCA CC-3') and sci-R (5'-TCT CTG CAG TCT GAA TGA CAT AAA CCT GCC GCT CC-3') for ahlS from S. sciuri. The SalI and PstI restriction sites were underlined in the primer sequences. PCR was performed with the following cycling parameters: $98^{\circ} \mathrm{C}$ for $10 \mathrm{~s}, 60{ }^{\circ} \mathrm{C}$ for $30 \mathrm{~s}$, and $68^{\circ} \mathrm{C}$ for $1 \mathrm{~min}$ for 30 cycles. The PCR products were digested by SalI and PstI and inserted into the same sites of a broad-host-range vector pBBR1MCS5 [25]. The prepared plasmids were transferred into E. coli $\mathrm{DH} 5 \alpha$ and used for AHL-degradation assays.

\subsection{Assessment of AHL-Degrading Activities}

The full-grown cultures of CNS stains or E. coli DH5 $\alpha$ harboring the AhlS-expressing plasmid were inoculated in fresh LB medium ( $1 \%$ inoculum) containing $100 \mu \mathrm{M}$ isopropyl- $\beta$-D-thiogalactopyranoside (IPTG). C6-HSL or C10-HSL was added at a final concentration of $20 \mu \mathrm{M}$ (for CNS strains) or $10 \mu \mathrm{M}$ (for E. coli). After incubation at $37^{\circ} \mathrm{C}$, cells were removed by centrifugation to obtain the supernatant. The residual AHLs in the supernatant were detected using C. violaceum CV026 and VIR07. An overnight culture of CV026 or VIR07 was mixed with $25 \mathrm{~mL} \mathrm{LB}$ agar medium and poured into Petri dishes. AHL samples $(20 \mu \mathrm{L})$ were placed in paper discs ( $8 \mathrm{~mm}$ in diameter, Advantec, Tokyo, Japan), which were subsequently placed onto LB agar plates containing CV026 or VIR07. Assay plates were incubated overnight at $30^{\circ} \mathrm{C}$ and the residual amounts of AHL were calculated using the relationship equations based on the size of the purple-colored zones and the known quantities of AHLs [26].

\subsection{AHL Restoration Assay}

The AHL-lactonase activity was assessed based on previously-established methods [27]. The full-grown culture of E. coli $\mathrm{DH} 5 \alpha$ harboring the AhlS-expressing plasmid was inoculated in $4 \mathrm{~mL}$ fresh LB medium (1\% inoculum) containing $100 \mu \mathrm{M}$ IPTG and $10 \mu \mathrm{M}$ C10-HSL. After incubation, the cells were removed by centrifugation at $12,000 \times g$ for $5 \mathrm{~min}$, and $90 \mu \mathrm{L}$ of the supernatant was mixed with $10 \mu \mathrm{L}$ of $1 \mathrm{~N} \mathrm{HCl}$. After incubation for $48 \mathrm{~h}$ at $4{ }^{\circ} \mathrm{C}, 20 \mu \mathrm{L}$ of $1 \mathrm{M}$ phosphate buffer (pH 7) was added to neutralize the $\mathrm{pH}$. The restored $\mathrm{C} 10-\mathrm{HSL}$ was detected with a VIR07 biosensor. 


\subsection{Purification of AhlS as an MBP Fusion}

The ahlS gene from S. sciuri ATCC 29060 was amplified with the KOD FX Neo system using the following set of primers: MBP-F (5'-TCT GGA TCC ATG GTA AAT GTA AAT AAC CAA GG-3') and MBP-R (5'-TCT CTG CAG TCT GAA TGA CAT AAA CCT GCC GCT CC-3'). The BamHI and PstI restriction sites were underlined in the primer sequences. PCR was performed with the following cycling parameters: $98^{\circ} \mathrm{C}$ for $10 \mathrm{~s}, 60^{\circ} \mathrm{C}$ for $30 \mathrm{~s}$, and $68^{\circ} \mathrm{C}$ for $1 \mathrm{~min}$ for 30 cycles. The PCR products were digested by BamHI and PstI and inserted into the same sites of a pMAL-c2X vector (New England Biolabs, Tokyo, Japan) to construct pMAL-ahlS.

The full-grown culture of $E$. coli DH5 $\alpha$ harboring pMAL-ahlS was inoculated into $100 \mathrm{~mL}$ fresh LB medium containing ampicillin. After incubation at $37^{\circ} \mathrm{C}$ for $2 \mathrm{~h}$, the expression of MBP-AhlS was induced by adding $1 \mathrm{mM} \mathrm{IPTG}$. After incubation for $18 \mathrm{~h}$ at $37^{\circ} \mathrm{C}$, cells were harvested by centrifugation and resuspended in the column buffer (20 mM Tris-HCl buffer and $200 \mathrm{mM} \mathrm{NaCl}, \mathrm{pH}$ 7.4). Lysozyme from egg white (Wako, Osaka, Japan) was added to the suspension at a final concentration of $250 \mu \mathrm{g} / \mathrm{mL}$. After incubation for $4 \mathrm{~min}$ at $37^{\circ} \mathrm{C}$, the suspension was sonicated and centrifuged to remove cell debris. The filtrated sample was loaded on an MBPTrap affinity chromatography column (GE Healthcare, Tokyo, Japan) equilibrated with the column buffer and eluted with $10 \mathrm{mM}$ maltose. As the negative control, MBP-LacZ $\alpha$ fusion was expressed and purified from cells of $E$. coli DH5 $\alpha$ harboring pMAL-c2X using the same method. The expression and purification of MBP fusion proteins were analyzed on $10 \%$ SDS-PAGE followed by Coomassie Brilliant Blue staining.

\subsection{Inhibition of Quorum Sensing by MBP-AhlS in P. aeruginosa}

P. aeruginosa PAO1 [28] and its quorum-sensing-deficient mutant PAO-MW1 [29] were incubated overnight at $37^{\circ} \mathrm{C}$ in the LB medium, and $40 \mu \mathrm{L}$ of each was inoculated into $4 \mathrm{~mL}$ PTSB medium [30] for the pyocyanin assay or $4 \mathrm{~mL} \mathrm{LB}$ medium for the elastase assay. The $200 \mu \mathrm{L}$ of AhlS solution (approximately $40 \mu \mathrm{g} / \mu \mathrm{L})$ or column buffer $(200 \mu \mathrm{L}$, the negative control) was added and incubated for $17 \mathrm{~h}$ at $37^{\circ} \mathrm{C}$. For the pyocyanin production assay, $450 \mu \mathrm{L}$ chloroform was mixed with $750 \mu \mathrm{L}$ of each culture supernatant and vortexed for $10 \mathrm{~s}$. The chloroform layer was transferred to a fresh tube, mixed with $150 \mu \mathrm{L} 0.2 \mathrm{M} \mathrm{HCl}$, and vortexed for $10 \mathrm{~s}$. After centrifugation, the absorbance of the aqueous layer was measured at $520 \mathrm{~nm}$. For the elastase assay, $10 \mathrm{mg}$ elastin-Congo red was added to $100 \mu \mathrm{L}$ of each culture supernatant, which was then mixed with $900 \mu \mathrm{L}$ of the assay buffer $(0.1 \mathrm{M}$ Tris-HCl, $1 \mathrm{mM}$ $\mathrm{CaCl}_{2}, \mathrm{pH}$ 8.0). After incubation for $4 \mathrm{~h}$ at $37^{\circ} \mathrm{C}$ with shaking at $1400 \mathrm{rpm}$, the precipitate was removed by centrifugation, and the absorbance was measured at $495 \mathrm{~nm}$. The relative pyocyanin production $\left(\mathrm{A}_{520} / \mathrm{OD}_{600}\right)$ and elastase activity $\left(\mathrm{A}_{495} / \mathrm{OD}_{600}\right)$ of PAO1-AhlS were calculated with those of PAO1LacZ $\alpha$ set to $100 \%$.

\subsection{Nucleotide Sequence Accession Numbers}

The nucleotide sequences of ahlS from S. sciuri ATCC 29060, 29061, and StLB252 have been deposited in the DDBJ/ENA/GenBank databases under the following accession number LC128625, LC128626, and LC128627, respectively.

\section{Conclusions}

In summary, our work is the first to report that ahlS from the CNS strains has AHL-degrading activity. It has been reported that CNS species are part of the normal flora on the skin and are less virulent. One of the CNS species, Staphylococcus epidermidis, is a normal colonizer of the skin and can be isolated from the diseased tissue alongside P. aeruginosa [31]. Interestingly, the extracellular substance secreted by $P$. aeruginosa affected the biofilm formation of $S$. epidermidis [31,32]. Since P. aeruginosa uses quorum sensing to regulate biofilm formation [33], it was assumed that the inhibition of quorum sensing by AhlS from the CNS strains was related to the niche competition against $P$. aeruginosa. Based on the results from the BLAST search, AhlS was also present in the genome of S. epidermidis 
NCTC 4133 (accession No. LR134242). Therefore, ahlS genes are assumed to be widely distributed among CNS strains. In this study, we used the purified AhlS protein for the quorum-quenching assay and demonstrated the inhibition of virulence factors, which are regulated by quorum sensing in P. aeruginosa. CNS strains might contribute to the inhibition of the quorum-sensing system of other pathogens in the normal flora of the skin. Meanwhile, it has not been elucidated whether CNS strains produce sufficient levels of AhlS for inhibition of quorum-sensing in other bacteria in natural systems, and the cell numbers of CNS strains are abundant enough within the community to impact the quorum-sensing system of more abundant microbes. Future studies could investigate how the behavior of AHL production and degradation on the surface of skins might contribute to elucidating the complicated quorum-sensing networks in the normal flora of the skin.

Supplementary Materials: The following are available online at http://www.mdpi.com/2079-6382/9/8/483/s1, Figure S1: Phylogenetic tree based on amino acid sequences of AhlS from CNS strains, Figure S2: Restoration of C10-HSL degraded by E. coli DH5 $\alpha$ harboring ahlS from CNS strains, Figure S3: SDS-PAGE analysis of MBP-AhlS.

Author Contributions: Conceptualization: T.M. and N.S.; methodology: Y.K. and T.M.; validation: Y.K. and T.M.; formal analysis: Y.K. and T.M.; investigation: Y.K. and T.M.; resources: N.S. and T.M.; data curation: Y.K. and T.M.; writing—original draft: T.M.; writing—review and editing: T.M.; visualization: Y.K. and T.M.; supervision: T.M. and N.S.; project administration: T.M.; funding acquisition: T.M. All authors have read and agreed to the published version of the manuscript.

Funding: This work was supported by the Grant-in-Aid for Young Scientists (B) (No. 24760643) from the Ministry of Education, Culture, Sports, Science and Technology of Japan.

Acknowledgments: We thank Ryusuke Sato and Takahito Yamaguchi from Utsunomiya University for technical assistance.

Conflicts of Interest: The authors declare no competing or financial interests.

\section{References}

1. Waters, C.M.; Bassler, B.L. Quorum sensing: Cell-to-cell communication in bacteria. Annu. Rev. Cell. Dev. Biol. 2005, 21, 319-346. [CrossRef] [PubMed]

2. Parsek, M.R.; Greenberg, E.P. Acyl-homoserine lactone quorum sensing in gram-negative bacteria: A signaling mechanism involved in associations with higher organisms. Proc. Nat. Acad. Sci. USA 2000, 97, 8789-8793. [CrossRef] [PubMed]

3. De Kievit, T.R.; Iglewski, B.H. Bacterial quorum sensing in pathogenic relationships. Infect. Immun. 2000, 68, 4839-4849. [CrossRef]

4. Von Bodman, S.B.; Bauer, W.D.; Coplin, D.L. Quorum sensing in plant-pathogenic bacteria. Annu. Rev. Phytopathol. 2003, 41, 455-482. [CrossRef] [PubMed]

5. Uroz, S.; Dessaux, Y.; Oger, P. Quorum sensing and quorum quenching: The yin and yang of bacterial communication. ChemBioChem 2009, 10, 205-216. [CrossRef] [PubMed]

6. Dong, Y.H.; Xu, J.L.; Li, X.Z.; Zhang, L.H. AiiA, an enzyme that inactivates the acylhomoserine lactone quorum-sensing signal and attenuates the virulence of Erwinia carotovora. Proc. Nat. Acad. Sci. USA 2000, 97, 3526-3531. [CrossRef] [PubMed]

7. Morohoshi, T.; Tominaga, Y.; Someya, N.; Ikeda, T. Complete genome sequence and characterization of the $\mathrm{N}$-acylhomoserine lactone-degrading gene of the potato leaf-associated Solibacillus silvestris. J. Biosci. Bioeng. 2012, 113, 20-25. [CrossRef]

8. Zhang, H.B.; Wang, L.H.; Zhang, L.H. Genetic control of quorum-sensing signal turnover in Agrobacterium tumefaciens. Proc. Nat. Acad. Sci. USA 2002, 99, 4638-4643. [CrossRef]

9. Park, S.Y.; Lee, S.J.; Oh, T.K.; Oh, J.W.; Koo, B.T.; Yum, D.Y.; Lee, J.K. AhlD, an N-acylhomoserine lactonase in Arthrobacter sp., and predicted homologues in other bacteria. Microbiology 2003, 149, 1541-1550. [CrossRef]

10. Wang, W.Z.; Morohoshi, T.; Someya, N.; Ikeda, T. AidC, a novel N-acylhomoserine lactonase from the potato root-associated Cytophaga-Flavobacteria-Bacteroides (CFB) group bacterium Chryseobacterium sp. strain StRB126. Appl. Environ. Microbiol. 2012, 78, 7985-7992. [CrossRef]

11. Morohoshi, T.; Tominaga, Y.; Someya, N.; Ikeda, T. Characterization of a novel thermostable $N$-acylhomoserine lactonase from the thermophilic bacterium Thermaerobacter marianensis. J. Biosci. Bioeng. 2015, 120, 1-5. [CrossRef] [PubMed] 
12. Huebner, J.; Goldmann, D.A. Coagulase-negative staphylococci: Role as pathogens. Annu. Rev. Med. 1999, 50, 223-236. [CrossRef] [PubMed]

13. McClean, K.H.; Winson, M.K.; Fish, L.; Taylor, A.; Chhabra, S.R.; Camara, M.; Daykin, M.; Lamb, J.H.; Swift, S.; Bycroft, B.W.; et al. Quorum sensing and Chromobacterium violaceum: Exploitation of violacein production and inhibition for the detection of $N$-acylhomoserine lactones. Microbiology 1997, 143, 3703-3711. [CrossRef]

14. Morohoshi, T.; Kato, M.; Fukamachi, K.; Kato, N.; Ikeda, T. N-acylhomoserine lactone regulates violacein production in Chromobacterium violaceum type strain ATCC 12472. FEMS Microbiol. Lett. 2008, 279, 124-130. [CrossRef]

15. Altschul, S.F.; Gish, W.; Miller, W.; Myers, E.W.; Lipman, D.J. Basic local alignment search tool. J. Mol. Biol. 1990, 215, 403-410. [CrossRef]

16. Neyaz, L.; Karki, A.B.; Fakhr, M.K. Draft genome sequence of megaplasmid-bearing Staphylococcus sciuri strain B9-58B, isolated from retail pork. Microbiol. Resour. Announc. 2020, 9, e01419-e01474. [CrossRef]

17. Morohoshi, T.; Someya, N.; Ikeda, T. Novel N-acylhomoserine lactone-degrading bacteria isolated from the leaf surface of Solanum tuberosum and their quorum-quenching properties. Biosci. Biotechnol. Biochem. 2009, 73, 2124-2127. [CrossRef]

18. Rumbaugh, K.P.; Griswold, J.A.; Iglewski, B.H.; Hamood, A.N. Contribution of quorum sensing to the virulence of Pseudomonas aeruginosa in vurn wound infections. Infect. Immun. 1999, 67, 5854-5862. [CrossRef]

19. Priyaja, P.; Jayesh, P.; Philip, R.; Bright Singh, I.S. Pyocyanin induced in vitro oxidative damage and its toxicity level in human, fish and insect cell lines for its selective biological applications. Cytotechnology 2016, 68, 143-155. [CrossRef]

20. Schleifer, K.H.; Fischer, U. Description of a new species of the genus Staphylococcus: Staphylococcus carnosus. Int. J. Syst. Evol. Microbiol. 1982, 32, 153-156. [CrossRef]

21. Schleifer, K.H.; Kloos, W.E. Isolation and characterization of Staphylococci from human skin I. amended descriptions of Staphylococcus epidermidis and Staphylococcus saprophyticus and descriptions of three new species: Staphylococcus cohnii, Staphylococcus haemolyticus and Staphylococcus xylosus. Int. J. Syst. Evol. Microbiol. 1975, 25, 50-61.

22. Kloos, W.E.; Schleifer, K.H.; Smith, R.F. Characterization of Staphylococcus sciuri sp. nov. and its subspecies. Int. J. Syst. Evol. Microbiol. 1976, 26, 22-37.

23. Sambrook, J. Molecular Cloning: A Laboratory Manual/Joseph Sambrook, David, W. Russell; Cold Spring Harbor Laboratory: Cold Spring Harbor, NY, USA, 2001.

24. Chhabra, S.R.; Harty, C.; Hooi, D.S.; Daykin, M.; Williams, P.; Telford, G.; Pritchard, D.I.; Bycroft, B.W. Synthetic analogues of the bacterial signal (quorum sensing) molecule $N$-(3-oxododecanoyl)-L-homoserine lactone as immune modulators. J. Med. Chem. 2003, 46, 97-104. [PubMed]

25. Kovach, M.E.; Elzer, P.H.; Hill, D.S.; Robertson, G.T.; Farris, M.A.; Roop, R.M., 2nd; Peterson, K.M. Four new derivatives of the broad-host-range cloning vector pBBR1MCS, carrying different antibiotic-resistance cassettes. Gene 1995, 166, 175-176. [CrossRef]

26. Ochiai, S.; Yasumoto, S.; Morohoshi, T.; Ikeda, T. AmiE, a novel N-acylhomoserine lactone acylase belonging to the amidase family, from the activated-sludge isolate Acinetobacter sp. strain Ooi24. Appl. Environ. Microbiol. 2014, 80, 6919-6925. [CrossRef]

27. Ochiai, S.; Morohoshi, T.; Kurabeishi, A.; Shinozaki, M.; Fujita, H.; Sawada, I.; Ikeda, T. Production and degradation of $\mathrm{N}$-acylhomoserine lactone quorum sensing signal molecules in bacteria isolated from activated sludge. Biosci. Biotechnol. Biochem. 2013, 77, 2436-2440. [CrossRef]

28. Holloway, B.W.; Krishnapillai, V.; Morgan, A.F. Chromosomal genetics of Pseudomonas. Microbiol. Rev. 1979, 43, 73-102. [CrossRef]

29. Whiteley, M.; Lee, K.M.; Greenberg, E.P. Identification of genes controlled by quorum sensing in Pseudomonas aeruginosa. Proc. Nat. Acad. Sci. USA 1999, 96, 13904-13909. [CrossRef]

30. Ishida, T.; Ikeda, T.; Takiguchi, N.; Kuroda, A.; Ohtake, H.; Kato, J. Inhibition of quorum sensing in Pseudomonas aeruginosa by $\mathrm{N}$-acyl cyclopentylamides. Appl. Environ. Microbiol. 2007, 73, 3183-3188. [CrossRef]

31. Pihl, M.; Chávez de Paz, L.E.; Schmidtchen, A.; Svensäter, G.; Davies, J.R. Effects of clinical isolates of Pseudomonas aeruginosa on Staphylococcus epidermidis biofilm formation. FEMS Immunol. Med. Microbiol. 2010, 59, 504-512. [CrossRef] 
32. Pihl, M.; Arvidsson, A.; Skepö, M.; Nilsson, M.; Givskov, M.; Tolker-Nielsen, T.; Svensäter, G.; Davies, J.R. Biofilm formation by Staphylococcus epidermidis on peritoneal dialysis catheters and the effects of extracellular products from Pseudomonas aeruginosa. Pathog. Dis. 2013, 67, 192-198. [CrossRef] [PubMed]

33. de Kievit, T.R. Quorum sensing in Pseudomonas aeruginosa biofilms. Environ. Microbiol. 2009, 11, $279-288$. [CrossRef] [PubMed]

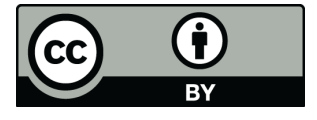

(C) 2020 by the authors. Licensee MDPI, Basel, Switzerland. This article is an open access article distributed under the terms and conditions of the Creative Commons Attribution (CC BY) license (http://creativecommons.org/licenses/by/4.0/). 